\title{
Periodic solutions for the $p$-Laplacian neutral functional differential system
}

\section{Zhenyou Wang and Changxiu Song*}

\section{"Correspondence:}

scx168@sohu.com

School of Applied Mathematics,

Guangdong University of

Technology, Guangzhou, 510006,

China

\begin{abstract}
By using the generalized Borsuk theorem in coincidence degree theory, we prove the existence of periodic solutions for the $p$-Laplacian neutral functional differential

system.

MSC: $34 C 25$

Keywords: $p$-Laplacian; periodic solutions; coincidence degree
\end{abstract}

\section{Introduction}

In recent years, the existence of periodic solutions for the Rayleigh equation and the Liénard equation has been studied (see [1-9]). By using topological degree theory, some results on the existence of periodic solutions are obtained.

Motivated by the works in [1-9], we consider the existence of periodic solutions of the following system:

$$
\frac{d}{d t} \phi_{p}\left[(x(t)-C x(t-\tau))^{\prime}\right]+\frac{d}{d t} \operatorname{grad} F(x(t))+\operatorname{grad} G(x(t))=e(t),
$$

where $F \in C^{2}\left(R^{n}, R\right), G \in C^{1}\left(R^{n}, R\right), e \in C\left(R, R^{n}\right)$ are periodic functions with period $T$; $C=$ $\left[c_{i j}\right]_{n \times n}$ is an $n \times n$ symmetric matrix of constants, $\tau \in R$ is a constant. $\phi_{p}: R^{n} \rightarrow R^{n}$ is given by

$$
\phi_{p}(u)=\phi_{p}\left(u_{1}, \ldots, u_{n}\right):=\left(\left|u_{1}\right|^{p-2} u_{1}, \ldots,\left|u_{n}\right|^{p-2} u_{n}\right)^{T}, \quad 1<p<\infty .
$$

The $\phi_{p}$ is a homeomorphism of $R^{n}$ with the inverse $\phi_{q}$. By using the theory of coincidence degree, we obtain some results to guarantee the existence of periodic solutions. Even for $p=2$, the results in this paper are also new.

In what follows, we use $\langle\cdot, \cdot\rangle$ to denote the Euclidean inner product in $R^{n}$ and $|\cdot|_{p}$ to denote the $l^{p}$-norm in $R^{n}$, i.e., $|x|_{p}=\left(\sum_{i=1}^{n}\left|x_{i}\right|^{p}\right)^{1 / p}$.

The norm in $R^{n \times n}$ is defined by $\|A\|_{p}=\sup _{|x|_{\rho}=1, x \in R^{n}}|A x|_{p}$.

The corresponding $L^{p}$-norm in $L^{p}\left([0, T], R^{n}\right)$ is defined by

$$
\|x\|_{p}=\left(\sum_{i=1}^{n} \int_{0}^{T}\left|x_{i}(t)\right|^{p} d t\right)^{\frac{1}{p}}=\left(\int_{0}^{T}|x(t)|_{p}^{p} d t\right)^{\frac{1}{p}},
$$

O2013 Wang and Song; licensee Springer. This is an Open Access article distributed under the terms of the Creative Commons Attribution License (http://creativecommons.org/licenses/by/2.0), which permits unrestricted use, distribution, and reproduction in any medium, provided the original work is properly cited. 
and the $L^{\infty}$-norm in $L^{\infty}\left([0, T], R^{n}\right)$ is

$$
\|x\|_{\infty}=\max _{1 \leq i \leq n}\left\|x_{i}\right\|_{\infty}
$$

where $\left\|x_{i}\right\|_{\infty}=\sup _{t \in[0, \omega]}\left|x_{i}(t)\right|(i=1, \ldots, n)$.

Let $W=W^{1, p}([0, T], R]$ be the Sobolev space.

Lemma 1.1 (See [8]) Suppose $u \in W$ and $u(0)=u(T)=0$, then

$$
\|u\|_{p} \leq\left(\frac{T}{\pi_{p}}\right)\left\|u^{\prime}\right\|_{p}
$$

where

$$
\pi_{p}=2 \int_{0}^{(p-1)^{1 / p}} \frac{d s}{\left(1-\frac{s^{p}}{p-1}\right)^{1 / p}}=\frac{2 \pi(p-1)^{1 / p}}{p \sin \left(\frac{\pi}{p}\right)} .
$$

In order to use coincidence degree theory to study the existence of $T$-periodic solutions for (1.1), we rewrite (1.1) in the following form:

$$
\left\{\begin{array}{l}
(x(t)-C x(t-\tau))^{\prime}(t)=\phi_{q}(y(t)), \\
y^{\prime}(t)=\frac{d}{d t} \operatorname{grad} F(x(t))-\operatorname{grad} G(x(t))+e(t) .
\end{array}\right.
$$

If $z(t)=\left(\begin{array}{l}x(t) \\ y(t)\end{array}\right)$ is a $T$-periodic solution of (1.2), x(t) must be a $T$-periodic solution of (1.1). Thus, the problem of finding a $T$-periodic solution for (1.1) reduces to finding one for (1.2). Let $C_{T}=\left\{x \in C\left(R, R^{n}\right): x(t+T) \equiv x(t)\right\}$ with the norm $\|x\|_{\infty}=\max _{1 \leq i \leq n}\left\|x_{i}\right\|_{\infty}, X=Z=$ $\left\{z=\left(\begin{array}{l}x(\cdot) \\ y(\cdot)\end{array}\right) \in C\left(R, R^{2 n}\right): z(t+T) \equiv z(t)\right\}$ with the norm $\|z\|=\max \left\{\|x\|_{\infty},\|y\|_{\infty}\right\}$. Clearly, $X$ and $Z$ are Banach spaces.

Denote the operator $A$ by

$$
A: C_{T} \rightarrow C_{T}, \quad(A x)(t)=x(t)-C x(t-\tau) .
$$

Meanwhile, let

$$
\begin{aligned}
& L: \operatorname{Dom} L \subset X \rightarrow Z, \quad(L z)(t)=z^{\prime}(t)=\left(\begin{array}{c}
(A x)^{\prime}(t) \\
y^{\prime}(t)
\end{array}\right), \\
& N: X \rightarrow Z, \\
& (N z)(t)=\left(\begin{array}{c}
\phi_{q}(y(t)) \\
-\frac{d}{d t} \operatorname{grad} F(x(t))-\operatorname{grad} G(x(t))+e(t)
\end{array}\right):=H(z, t) .
\end{aligned}
$$

It is easy to see that $\operatorname{Ker} L=R^{2 n}, \operatorname{Im} L=\left\{z \in Z: \int_{0}^{T} z(s) d s=0\right\}$. So, $L$ is a Fredholm operator with index zero. Let $P: X \rightarrow \operatorname{Ker} L$ and $Q: Z \rightarrow \operatorname{Im} Q$ be defined by

$$
\begin{aligned}
P u & =\frac{1}{T} \int_{0}^{T} u(s) d s, \quad u \in X ; \\
Q v & =\frac{1}{T} \int_{0}^{T} v(s) d s, \quad v \in Z,
\end{aligned}
$$

and let $K_{p}$ denote the inverse of $\left.L\right|_{\operatorname{Ker} P \cap \operatorname{Dom} L}$. 
Obviously, $\operatorname{Ker} L=\operatorname{Im} Q=R^{2 n}$ and

$$
\left(K_{p} z\right)(t)=\left(\begin{array}{c}
\left(A^{-1} F x\right)(t) \\
(F y)(t)
\end{array}\right)
$$

where $z=\left(x^{T}(\cdot), y^{T}(\cdot)\right)^{T} \in Z,(F h)(t)=\int_{0}^{t} h(s) d s-\frac{1}{T} \int_{0}^{T} \int_{0}^{t} h(s) d s d t, h \in C_{T}$.

From (1.3), one can easily see that $N$ is $L$-compact on $\bar{\Omega}$, where $\Omega$ is an open bounded subset of $X$.

Lemma 1.2 (See [9]) Suppose that $\lambda_{1}, \lambda_{2}, \ldots, \lambda_{n}$ are eigenvalues of the matrix C. If $\left|\lambda_{i}\right| \neq 1$, $\forall i \in\{1,2, \ldots, n\}$, then $A$ has a continuous bounded inverse $A^{-1}$ with the following relationships:

(1) $\left\|A^{-1} u\right\|_{\infty} \leq\left(\sum_{i=1}^{n} \frac{1}{|1-| \lambda_{i}||}\right)\|u\| \infty, \forall u \in C_{T}$;

(2) $\left\|A^{-1} u\right\|_{p}^{p} d t \leq \sigma\|u\|_{p}^{p} d t, \forall u \in C_{T}, p \geq 1$, where

$$
\sigma= \begin{cases}\max _{i \in\{1,2, \ldots, n\}}\left\{\frac{1}{|1-| \lambda_{i}||^{2}}\right\}, & p=2, \\ \left(\sum_{i=1}^{n} \frac{1}{|1-| \lambda_{i}||^{2 p}}\right)^{(2-p) / 2}, & p \in[1,2), \\ \left(\sum_{i=1}^{n} \frac{1}{|1-| \lambda_{i}||^{q}}\right)^{p / q}, & p \in(2,+\infty),\end{cases}
$$

where $q$ is a constant with $1 / p+1 / q=1$;

(3) $A x^{\prime}=(A x)^{\prime}, \forall x \in C_{T}^{\prime}$.

In the proof of our results on the existence of periodic solutions, we use the following generalized Borsuk theorem in coincidence degree theory of Gaines and Mawhin [10].

Lemma 1.3 Let $X$ and $Z$ be real normed vector spaces. Let $L$ be a Fredholm mapping of index zero. $\Omega$ is an open bounded subset of $X$ and $\Omega$ is symmetric with respect to the origin and contains it. Let $\tilde{N}: \bar{\Omega} \times[0,1] \rightarrow Z$ be L-compact and such that

(a) $\tilde{N}(-x, 0)=-\tilde{N}(x, 0), \forall x \in \bar{\Omega}$,

(b) $L x \neq \tilde{N}(x, \lambda), \forall x \in \operatorname{Dom} L \cap \partial \Omega$.

Then, for every $\lambda \in[0,1]$, the equation $L x=\tilde{N}(x, \lambda)$ has at least one solution in $\Omega$.

\section{Main results}

Theorem 2.1 Suppose that the matrix $C$ satisfies the conditions of Lemma 1.2 and that there exist constants $a>0, b>0, c \geq 0$ and $\alpha>1$ such that

(H1) $y^{T} \frac{\partial^{2} F(x)}{\partial x^{2}} y \geq a|y|_{2}^{2}$ or $y^{T} \frac{\partial^{2} F(x)}{\partial x^{2}} y \leq-a|y|_{2}^{2}, \forall x, y \in R^{n}$;

(H2) $\langle y, \operatorname{grad} G(x)\rangle \geq b|y|_{\alpha}^{\alpha}-c, \forall x, y \in R^{n}$.

Then equation (1.1) has at least one T-periodic solution for $1<p \leq 2$.

Proof For any $\lambda \in[0,1]$, let

$$
\tilde{N}(z, \lambda)(t)=\frac{1+\lambda}{2} H(z, t)-\frac{1-\lambda}{2} H(-z, t) .
$$

Consider the following parameter equation:

$$
(L z)(t)=\tilde{N}(z, \lambda)(t), \quad \lambda \in[0,1] .
$$


Let $z(t)=\left(\begin{array}{l}x(t) \\ y(t)\end{array}\right)$ be a possible $T$-periodic solution of (2.3) for some $\lambda \in[0,1]$, then $x=x(t)$ is a $T$-periodic solution of the following system:

$$
\begin{gathered}
\left(\phi_{p}\left(\left(A x^{\prime}\right)(t)\right)\right)^{\prime}+\frac{1+\lambda}{2} \frac{d}{d t} \operatorname{grad} F(x(t))-\frac{1-\lambda}{2} \frac{d}{d t} \operatorname{grad} F(-x(t)) \\
+\frac{1+\lambda}{2} \operatorname{grad} G(x(t))-\frac{1-\lambda}{2} \operatorname{grad} G(-x(t))=\lambda e(t) .
\end{gathered}
$$

Noticing that $x(t)$ is a $T$-periodic solution, we have

$$
-\left\|A x^{\prime}\right\|_{p}^{p}=\int_{0}^{T}\left\langle A x,\left(\phi_{p}\left(A x^{\prime}\right)\right)^{\prime}\right\rangle d t
$$

Multiplying the two sides of $(2.2)$ by $(A x)(t)$ and integrating them on the interval $[0, T]$, by $(2.3)$ and $(\mathrm{H} 1)-(\mathrm{H} 2)$, we obtain

$$
-\left\|A x^{\prime}\right\|_{p}^{p}+a\|A x\|_{2}^{2}+b\|A x\|_{\alpha}^{\alpha}-c T \leq\|e\|_{\beta}\|A x\|_{\alpha}, \quad \text { where } \frac{1}{\alpha}+\frac{1}{\beta}=1 .
$$

On the other hand,

$$
\int_{0}^{T}\left\langle A x^{\prime},\left[\phi_{p}\left(A x^{\prime}\right)\right]^{\prime}\right\rangle d t=0 .
$$

So, multiplying the two sides of (2.2) by $\left(A x^{\prime}\right)(t)$ and integrating them on the interval $[0, T]$, by $(\mathrm{H} 1)-(\mathrm{H} 2)$, we get

$$
a\left\|A x^{\prime}\right\|_{2}^{2}-c T \leq a\|A x\|_{2}^{2}+b\|A x\|_{\alpha}^{\alpha}-c T \leq\|e\|_{2}\left\|A x^{\prime}\right\|_{2} .
$$

Furthermore, we have

$$
\left\|A x^{\prime}\right\|_{2} \leq \sqrt{\frac{c T}{a}+\frac{\|e\|_{2}^{2}}{4 a^{2}}}+\frac{\|e\|_{2}}{2 a}:=R_{1} .
$$

It is obvious that there exist $c_{1}>0$ and $c_{2}>0$ such that

$$
c_{1}|x|_{2} \leq|x|_{p} \leq c_{2}|x|_{2}, \quad x \in R^{n} .
$$

Thus,

$$
\begin{aligned}
\left\|A x^{\prime}\right\|_{p}^{p} & =\int_{0}^{T}\left|\left(A x^{\prime}\right)(t)\right|_{p}^{p} d t \\
& \leq c_{2}^{p}\left(\int_{0}^{T}\left|A x^{\prime}(t)\right|_{2}^{2} d t\right)^{p / 2} T^{(2-p) / 2} \\
& \leq\left(c_{2} R_{1}\right)^{p} T^{(2-p) / 2}:=R_{2},
\end{aligned}
$$

where $1<p \leq 2$.

From (2.4) and (2.5), we can see

$$
b\|A x\|_{\alpha}^{\alpha}-\|e\|_{\beta}\|A x\|_{\alpha}-c T \leq R_{2}-a\left\|A x^{\prime}\right\|_{2}^{2} \leq R_{2},
$$


from which it follows that there exists a positive number $R_{3}$ such that

$$
\|A x\|_{\alpha} \leq R_{3} .
$$

By using Lemma 1.2, we get

$$
\begin{aligned}
\|x\|_{\alpha} & =\left(\int_{0}^{T}|x(t)|_{\alpha}^{\alpha} d t\right)^{1 / \alpha} \\
& =\left(\int_{0}^{T}\left|A^{-1}(A x)(t)\right|_{\alpha}^{\alpha} d t\right)^{1 / \alpha} \\
& \leq \sigma^{1 / \alpha}\left(\int_{0}^{T}|(A x)(t)|_{\alpha}^{\alpha} d t\right)^{1 / \alpha} \\
& \leq \sigma^{1 / \alpha} R_{3}:=R_{4} .
\end{aligned}
$$

From (2.6), there exists $t_{0} \in[0, T)$ such that $\left|x\left(t_{0}\right)\right|_{\alpha} \leq R_{4} T^{-1 / \alpha}$, and

$$
\begin{aligned}
\left|x_{i}(t)\right| & =\left|x_{i}\left(t_{0}\right)+\int_{t_{0}}^{t} x_{i}^{\prime}(s) d s\right| \\
& \leq R_{4} T^{-1 / \alpha}+\sqrt{T}\left(\int_{0}^{T}\left(x_{i}^{\prime}(s)\right)^{2} d s\right)^{1 / 2} \\
& \leq R_{4} T^{-1 / \alpha}+\sqrt{T} R_{1}:=R_{5} .
\end{aligned}
$$

Therefore $\|x\|_{\infty} \leq R_{5}$ and $|x(t)|_{p} \leq n^{1 / p} R_{5}$.

Since $F \in C^{2}\left(R^{n}, R\right), G \in C^{1}\left(R^{n}, R\right)$, there exist $R_{6}$ and $R_{7}$ such that

$$
\left\|\frac{\partial^{2} F(x)}{\partial x^{2}}\right\|_{p} \leq R_{6}, \quad|\operatorname{grad} G(x)|_{p} \leq R_{7} \quad \text { for }|x|_{p} \leq n^{1 / p} R_{5} .
$$

From (2.4), we have

$$
\begin{aligned}
\int_{0}^{T}\left|\left(\phi_{p}\left(A x^{\prime}\right)\right)^{\prime}\right|_{p} d t & \leq R_{6} \int_{0}^{T}\left|x^{\prime}\right|_{p} d t+R_{7} T+\int_{0}^{T}|e(t)|_{p} d t \\
& \leq R_{6} T^{1 / q}\left\|x^{\prime}\right\|_{p}+R_{7} T+\int_{0}^{T}|e(t)|_{p} d t \\
& \leq R_{6} T^{1 / q} R_{2}^{1 / p}+R_{7} T+\int_{0}^{T}|e(t)|_{p} d t:=R_{8} .
\end{aligned}
$$

Clearly, for each $i=1, \ldots, n$, there exists $t_{i} \in(0, T)$ such that $x_{i}^{\prime}\left(t_{i}\right)=0$. Thus, for any $t \in[0, T]$, we have

$$
\begin{aligned}
\left|y_{i}(t)\right| & =\left|\phi_{p}\left(\left(A x_{i}\right)^{\prime}(t)\right)\right| \\
& =\left|\phi_{p}\left(\left(A x_{i}\right)^{\prime}(t)\right)-\phi_{p}\left(\left(A x_{i}\right)^{\prime}\left(t_{i}\right)\right)\right| \\
& =\left|\int_{t_{i}}^{t}\left(\phi_{p}\left(\left(A x_{i}\right)^{\prime}(s)\right)\right)^{\prime} d s\right| \\
& \leq R_{8} .
\end{aligned}
$$


Therefore $\|y\|_{\infty} \leq R_{8}$.

Choose a number $R_{9}>\max \left(R_{5}, R_{8}\right)$, and let $\Omega=\left\{z \in X:\|z\|<R_{9}\right\}$, then $L z \neq \tilde{N}(z, \lambda)$ for any $z \in \operatorname{Dom} L \cap \partial \Omega, \lambda \in[0,1]$. It is easy to see that $\tilde{N}$ is $L$-compact on $\bar{\Omega} \times[0,1], L z=\tilde{N}(z, 1)$ is $(2.1)$ and $\tilde{N}(-z, 0)=-\tilde{N}(z, 0)$. From Lemma 1.3, (2.1) has at least one $T$-periodic solution $\tilde{z}=\left(\begin{array}{l}\tilde{x}(t) \\ \tilde{y}(t)\end{array}\right), \tilde{x}(t)$ is a $T$-periodic solution of (1.1).

Theorem 2.2 Let $\lambda_{\infty}=\max \left\{\left|\lambda_{1}\right|,\left|\lambda_{2}\right|, \ldots,\left|\lambda_{n}\right|\right\}$, where $\lambda_{1}, \lambda_{2}, \ldots, \lambda_{n}$ are eigenvalues of the matrix $C$ with $\left|\lambda_{i}\right| \neq 1, \forall i \in\{1,2, \ldots, n\}$. Suppose that there exist constants $b \geq 0, c \geq 0$ and $d>0$ such that

(H3) there is a constant $r \geq 0$ such that $\lim _{|x| \rightarrow+\infty} \frac{|\operatorname{grad} F(x)|}{|x|^{p-1}} \leq r$;

(H4) $\langle y, \operatorname{grad} G(x)\rangle \leq b|y|_{p}^{p}+c, \forall x, y \in R^{n}$;

(H5) $\forall i \in\{1, \ldots, n\}$, either $x_{i}\left[\frac{\partial G(x)}{\partial x_{i}}-\bar{e}_{i}\right]>0$ or $x_{i}\left[\frac{\partial G(x)}{\partial x_{i}}-\bar{e}_{i}\right]<0$ for $\left|x_{i}\right|>d$, where $\bar{e}_{i}=\frac{1}{T} \int_{0}^{T} e_{i}(t) d t$.

Then (1.1) has at least one T-periodic solution for $\left(\lambda_{\infty} r+b\right) \frac{T}{\pi_{p}}<\sigma$.

Proof Let $z(t)=\left(\begin{array}{l}x(t) \\ y(t)\end{array}\right)$ be a possible $T$-periodic solution of (2.1). From assumption (H3), there exists a constant $\rho>d$ such that

$$
|\operatorname{grad} F(x)|<r|x|^{p-1}, \quad \forall x \in R^{n} \text { with }\left|x_{i}\right|>\rho \text { for } i=1,2, \ldots, n .
$$

From (H3) and (2.2), we have

$$
\begin{aligned}
& -\left\|A x^{\prime}\right\|_{p}^{p}+\int_{0}^{T}\left\langle A x(t), \frac{1+\lambda}{2} \frac{d}{d t} \operatorname{grad} F(x(t))-\frac{1-\lambda}{2} \frac{d}{d t} \operatorname{grad} F(-x(t))\right\rangle d t+b\|x\|_{p}^{p}+c T \\
& \quad \geq \lambda \int_{0}^{T}\langle x(t), e(t)\rangle d t \geq-\|e\|_{q}\|x\|_{p},
\end{aligned}
$$

i.e.,

$$
\begin{aligned}
\left\|A x^{\prime}\right\|_{p}^{p} \leq & \int_{0}^{T}\left\langle C x^{\prime}(t-\tau), \frac{1+\lambda}{2} \frac{d}{d t} \operatorname{grad} F(x(t))-\frac{1-\lambda}{2} \frac{d}{d t} \operatorname{grad} F(-x(t))\right\rangle d t \\
& +b\|x\|_{p}^{p}+\|e\|_{q}\|x\|_{p}+c T \\
\leq & \left\|C x^{\prime}\right\|_{p}\|\operatorname{grad} F(x(t))\|_{\frac{p}{p-1}}+b\|x\|_{p}^{p}+\|e\|_{q}\|x\|_{p}+c T \\
\leq & \lambda \infty\left\|x^{\prime}\right\|_{p}\left(r\|x\|_{p}^{p-1}+\theta\right)+b\|x\|_{p}^{p}+\|e\|_{q}\|x\|_{p}+c T,
\end{aligned}
$$

where $\theta=\max _{|u| \leq \sqrt{n} p}|\operatorname{grad} F(u)| T^{(p-1) / p}$.

Integrating both sides of $(2.2)$ over $[0, T]$, we get

$$
\frac{1+\lambda}{2} \int_{0}^{T}\left[\frac{\partial G(x(t))}{\partial x_{i}}-\bar{e}_{i}\right] d t-\frac{1-\lambda}{2} \int_{0}^{T}\left[\frac{\partial G(-x(t))}{\partial x_{i}}-\bar{e}_{i}\right] d t=0, \quad i=1, \ldots, n .
$$

So, there exist $\tilde{t}_{i} \in[0, T]$ such that

$$
\frac{1+\lambda}{2} \int_{0}^{T}\left[\frac{\partial G\left(x\left(\tilde{t}_{i}\right)\right)}{\partial x_{i}}-\bar{e}_{i}\right] d t-\frac{1-\lambda}{2} \int_{0}^{T}\left[\frac{\partial G\left(-x\left(\tilde{t}_{i}\right)\right)}{\partial x_{i}}-\bar{e}_{i}\right] d t=0, \quad i=1, \ldots, n .
$$


From (H4), one can see $\left|x_{i}\left(\tilde{t}_{i}\right)\right| \leq d$. Let $\chi_{i}(t)=x_{i}\left(t+\tilde{t}_{i}\right)-x_{i}\left(\tilde{t}_{i}\right), \chi(t)=\left(\chi_{1}(t), \ldots, \chi_{n}(t)\right)^{T}$, then $\chi(0)=\chi(T)=0$. By Lemma 1.1, one can obtain

$$
\|\chi\|_{p} \leq \frac{T}{\pi_{p}}\left\|\chi^{\prime}\right\| p
$$

Noticing the periodicity of $x(t)$, we have

$$
\begin{aligned}
\left\|x_{i}\right\|_{p}^{p} & =\int_{0}^{T}\left|x_{i}(t)\right|^{p} d t \\
& =\int_{0}^{T}\left|x_{i}\left(t+\tilde{t}_{i}\right)\right|^{p} d t \\
& \leq \int_{0}^{T}\left(\left|\chi_{i}(t)\right|+d\right)^{p} d t \\
& \leq\left(\left\|\chi_{i}\right\|_{p}+T^{1 / p} d\right)^{p} .
\end{aligned}
$$

From Minkovski's inequality, we have

$$
\begin{aligned}
\|x\|_{p} & =\left(\sum_{i=1}^{n}\left\|x_{i}\right\|_{p}^{p}\right)^{1 / p} \\
& \leq\left(\sum_{i=1}^{n}\left\|\chi_{i}(t)\right\|+T^{1 / p} d\right)^{p} \\
& \leq\|\chi\|_{p}+(n T)^{1 / p} d \leq \frac{T}{\pi_{p}}\left\|\chi^{\prime}\right\|_{p}+(n T)^{1 / p} d \\
& =\frac{T}{\pi_{p}}\left\|x^{\prime}\right\|_{p}+(n T)^{1 / p} d .
\end{aligned}
$$

In view of (2.7) and Lemma 1.2, we get

$$
\begin{aligned}
\sigma\left\|x^{\prime}\right\|_{p}^{p} \leq & \lambda_{\infty}\left\|x^{\prime}\right\|_{p}\left(r\left(\frac{T}{\pi_{p}}\left\|x^{\prime}\right\|_{p}+(n T)^{1 / p} d\right)^{p-1}+\theta\right) \\
& +b\left(\frac{T}{\pi_{p}}\left\|x^{\prime}\right\|_{p}+(n T)^{1 / p} d\right)^{p}+\|e\|_{q}\left(\frac{T}{\pi_{p}}\left\|x^{\prime}\right\|_{p}+(n T)^{1 / p} d\right)+c T .
\end{aligned}
$$

Since $\left(\lambda_{\infty} r+b\right) \frac{T}{\pi_{p}}<\sigma$, from (2.8), there exists a constant $R_{9}>0$ such that

$$
\left\|x^{\prime}\right\|_{p} \leq R_{9}
$$

Therefore,

$$
\|x\|_{p} \leq \frac{T}{\pi_{p}} R_{9}+(n T)^{1 / p} d:=R_{10} .
$$

From (2.9) and (2.10), we know that the rest of the proof of the theorem is similar to that of Theorem 2.1. 
Remark 2.1 If $C \equiv \mathbf{0}_{n \times n}$, system (1.1) can be reduced to the system in [2].

If $C \equiv \mathbf{0}_{n \times n}$ and $p=2$, system (1.1) can be reduced to the system in [3].

Example 2.1 Consider the following system:

$$
\frac{d}{d t} \phi_{p}\left[(x(t)-C x(t-\tau))^{\prime}\right]+\frac{d}{d t} \operatorname{grad} F(x(t))+\operatorname{grad} G(x(t))=e(t),
$$

where $F \in C^{2}\left(R^{2}, R\right), G \in C^{1}\left(R^{2}, R\right), e \in C\left(R, R^{2}\right)$ are periodic functions with period $T$; $C=$ $\left(\begin{array}{cc}-1 & -1 \\ -1 & 0\end{array}\right)$. Clearly, $\lambda_{1,2}=\frac{1 \pm \sqrt{5}}{2} \neq \pm 1$.

Let

$$
x=\left(x_{1}, x_{2}\right)^{T}, \quad F\left(x_{1}, x_{2}\right)=x_{1}^{2}+x_{2}^{2}-\frac{x_{1} x_{2}}{2}, \quad G\left(x_{1}, x_{2}\right)=x_{1}^{4}+x_{1}^{3}-\frac{1}{4} x_{1}^{2} x_{2}^{2}+x_{2}^{4},
$$

then, by Theorem 2.1, (2.11) has at least one $T$-periodic solution for $1<p \leq 2$.

\section{Competing interests}

The authors declare that they have no competing interests.

\section{Authors' contributions}

The first author carried out the studies, the second author participated in the studies and drafted the manuscript. All authors read and approved the final manuscript.

\section{Acknowledgements}

The authors thank the referees for helpful suggestions. This work was supported by Grants Nos. 10871052 and 109010600 from NNSF of China, and by Grant No. S2011010005029 from NSF of Guangdong.

Received: 13 May 2013 Accepted: 27 September 2013 Published: 13 Dec 2013

\section{References}

1. Din, T, lannacci, R, Zanolin, F: Existence and multiplicity results for periodic solutions of semilinear Duffing equations. J. Differ. Equ. 105, 364-409 (1993)

2. Peng, S, Xu, Z: On the existence of periodic solutions for a class of $p$-Laplacian system. J. Math. Anal. Appl. 325 , 166-174 (2007)

3. Ge, W: On the existence of harmonic solutions of Liénard system. Nonlinear Anal. 16, 183-190 (1991)

4. Manásevich, R, Mawhin, J: Periodic solutions for nonlinear systems with $p$-Laplacian-like operators. J. Differ. Equ. 145, 367-393 (1998)

5. Mawhin, J: Some boundary value problems for Hartman-type perturbations of the ordinary vector $p$-Laplacian. Nonlinear Anal. 40, 497-503 (1998)

6. Peng, S, Zhu, S: Periodic solutions for a Rayleigh system of p-Laplacian type. Nonlinear Anal. 70, 274-279 (2009)

7. Lu, S, Ge, W: Periodic solutions for a kind of Liénard equation with a deviating argument. J. Math. Anal. Appl. 289, 231-243 (2004)

8. Zhang, MR: Nonuniform nonresonance at the first eigenvalue of the $p$-Laplacian. Nonlinear Anal. 29, 41-51 (1997)

9. Lu, S: Periodic solutions to a second order $p$-Laplacian neutral functional differential system. Nonlinear Anal. 69, 4215-4229 (2008)

10. Gaines, RE, Mawhin, JL: Coincidence Degree and Nonlinear Differential Equations. Springer, Berlin (1977)

10.1186/1687-1847-2013-367

Cite this article as: Wang and Song: Periodic solutions for the $p$-Laplacian neutral functional differential system. Advances in Difference Equations 2013, 2013:367 